\title{
Preparation of Photo-cured Hybrid Thin Films using Zirconia Nanoparticles Modified with Dual Site Silane Coupling Agent
}

\author{
Yuki Minami ${ }^{\mathrm{a}, \mathrm{b}}$, Kazunori Murata ${ }^{\mathrm{b}}$, Seiji Watase ${ }^{\mathrm{c}}$, \\ and Kimihiro Matsukawa*a,c \\ ${ }^{a}$ Osaka City University, 3-3-138 Sugimoto, Sumiyoshi-ku, Osaka 558-8585, Japan \\ ${ }^{b}$ SOLAR CO., Ltd. , 1174-1 Nakano-cho, Kasai-shi, Hyogo 675-2102, Japan \\ ${ }^{c}$ Osaka Municipal Technical Research Institute, 1-6-50, Morinomiya, Joto-ku, Osaka 536-8553, Japan \\ kmatsu@omtri.or.jp
}

Keywords: zirconia nanoparticles, dispersion, high refractive index, hybrid thin film

\section{Introduction}

High refractive index material contributes to power saving and high performance of electronic devices which has been developed rapidly in recent years. However, organic materials by themselves cannot reach to the physical properties for using at practical optical device. In this point of view, it has been reported that high refractive index thin films could prepare from the organic-inorganic hybrid by the sol-gel method including inorganic component derived from metal alkoxide [1,2]. On the other hand, the optimum dispersant for nanoparticles is necessary for the accomplishment of intended properties avoiding the immiscibility with matrix [3]. We have already developed the dual site silane coupling agent with bisphenylfluorene and reported that it is effective for surface treatment of zirconia nanoparticle [4-6]. In this paper, we synthesized novel dual site silane coupling agent having phthalic acid ester and studied the preparation of zirconia nanoparticles dispersion. And also, the preparation of hybrid thin films with photo-cured polymers and their optical properties were studied.

\section{Experimental}

Dual site silane coupling agent, $o$-DAP-Si, containing phthalic acid ester was prepared by thiol-ene reaction of $o$-diallyl phthalate (o-DAP) and mercaptopropyl trimethoxysilane (MPTMS) (Scheme 1). This reaction was proceeded with a photo radical initiator, Irgacure 1173 (2-Hydroxy2-methyl-1-phenyl-propan-1-one). The mixture was reacted under UV irradiation $\left(1610 \mathrm{~mJ} / \mathrm{cm}^{2}\right)$ at cooling in an icewater bath. Zirconia nanoparticles dispersion was prepared from $5 \mathrm{wt} \%$ aggregate of zirconia nanoparticles (primary particle size: $10-20 \mathrm{~nm}$ ) and $o$-DAP in MEK by treatment of the bead milling using $50 \mu \mathrm{m}$ media beads. Zirconia nanoparticle dispersion obtained<smiles>C=CCOC(=O)c1ccccc1C(=O)OCC=C</smiles>

Scheme 1 
by this way was abbreviated as $o-\mathrm{DAP}-\mathrm{Si} / \mathrm{ZrO}_{2}$. High refractive index hybrid thin films were prepared from the mixture of $o-\mathrm{DAP}-\mathrm{Si} / \mathrm{ZrO} \mathrm{O}_{2}$ and multifunctional acrylates with a photo radical initiator. These coating solutions with various composition ratios of zirconia and monomers were spincoated on glass substrates. Transparent photo-cured thin films were obtained by UV irradiation after drying at $80{ }^{\circ} \mathrm{C}$ for $3 \mathrm{~min}$.

\section{Results and Discussion}

As a dual site silane coupling agent such as oDAP-Si has two trialkoxysilyl groups at the same side of molecule, it is seemed that alkoxysilyl groups are easily reacted with the surface of zirconia nanoparticles compared to the conventional silane coupling agent. Therefore, it can be expected more efficient dispersing of zirconia nanoparticles and modifying surface with phthalic acid ester, which provide a wide range of compatibility with multifunctional acrylate. The dispersed particle size was measured by DLS analysis, which was estimated as $20-30 \mathrm{~nm}$, and it was also confirmed by TEM observation.

The hybrid thin films of DPHA and $o$-DAP$\mathrm{Si} / \mathrm{ZrO}_{2}$ was transparent comparable to the simple photo-cured multifunctional acrylate, which were over $90 \%$ transmittance and less 1\% HAZE, even if increasing particulate concentration in the film. It was revealed that phthalic acid ester on the surface of nanoparticles indicated a function of plasticizer for good compatibility with monomers and polymers. The refractive index of hybrid thin films was increased to 1.70 from 1.52 with increasing of $o$-DAP-Si/ZrO ${ }_{2}$ (Figure 1). And PETA also can apply to the preparation of high refractive index hybrid thin films (Figure 2). These films showed the same tendency of refractive index with contents of $\mathrm{ZrO}_{2}$.

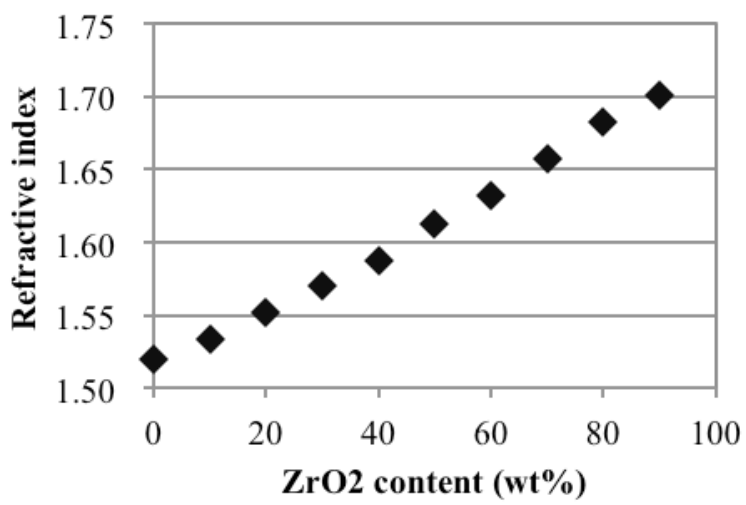

Figure 1 Refractive index of hybrid thin films of DPHA and $\mathrm{ZrO}_{2} / o$-DAP-Si depend on modified zirconia nanoparticle.

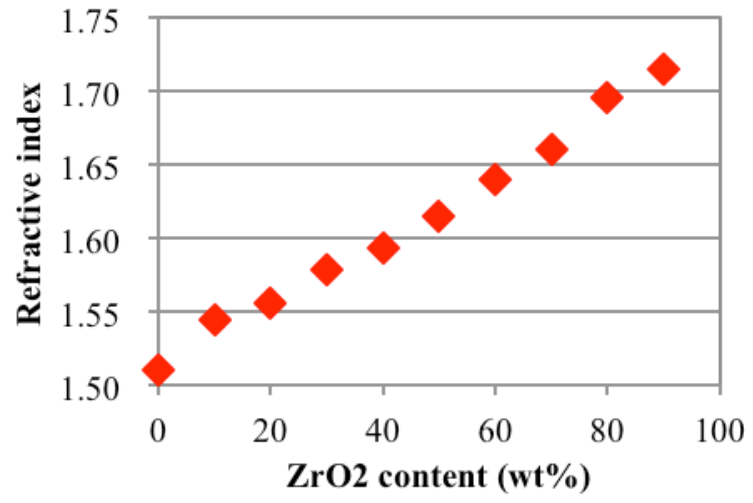

Figure 2 Refractive index of hybrid thin films of PETA and $\mathrm{ZrO}_{2} / o$-DAP-Si depend on modified zirconia nanoparticle.

\section{Conclusion}

The dual site silane coupling agent was synthesized by thiol-ene reaction. Zirconia nanoparticles could be dispersed effectively by using it. Surface bonded phtalic acid esters acted as a plasticizer to provide a good compatibility with photo-curable acrylates. High refractive index hybrid thin film indicated high transparency even though high nanoparticle contents. Therefore, hybrid thin films with photo-cured acrylates are possible to control their refractive index, and they will be expected to apply to optical materials.

\section{Acknowledgment}

This work was supported by a Grant-in-Aid for Scientific Research on Innovative Areas "New Polymeric Materials Based on Element-Blocks (No. 2401)" (24102010) of The Ministry of Education, Culture, Sports, Science, and Technology, Japan

\section{References}

1. H. I. Elim, Y. Kurata, O. Sugihara, T. Kaino, T. Adschiri, A-Li. Chu, and N. Kambe, J. Phys. Chem. B, 113 (2009), 10143.

2. Y. Imai, A. Terahara, Y. Hakuta, K. Matsui, H. Hayashi, and N. Ueno, Eur. Poly. Journal, 45 (2009), 630.

3. Y. Minami, K. Murata, S. Watase, K. Matsukawa, Koubunshi Ronbunshu, 67 (2010) 397.

4. K. Matsukawa, Y. Matsuura, A. Nakamura, N. Nishioka, T. Motokawa, and H. Murase, $J$. Photopolym. Sci. Technol., 19 (2006), 89.

5. K. Matsukawa, Y. Matsuura, A. Nakamura, N. Nishioka, H. Murase, and S. Kawasaki, J. Photopolym. Sci. Technol., 20 (2007), 307.

6. Y. Minami, K. Murata, S. Watase, A. Matsumoto, and K. Matsukawa, J. Photopolym. Sci. Technol., 26 (2013), 491. 